\title{
Modelling Stochastic Calcium Waves in Cardiac Myocytes Based on the Two- Pool CICR Model
}

\author{
Serife Arif, Choi-Hong Lai, Nadarajah I Ramesh \\ Mathematical Sciences Department, Numerical and Applied Mathematics Research Unit \\ University of Greenwich, London, UK
}

\begin{abstract}
In this paper, stochastic $1 D$ and $2 D$ models based on the two-pool calcium-induced calcium release (CICR) process are presented. An anisotropic diffusion model is presented that approximates the calcium concentration in the cytosol as well as the sarcoplasmic reticulum (SR).

The relative source strengths in $1 D$ and $2 D$ are adjusted to simulate the behaviour of a point source array in the real $3 D$ problem. The numerical conversion presented involves the convolution between the Green's and Heaviside functions. The models are solved using the Crank Nicolson $(C N)$ and alternating direction implicit (ADI) for the $I D$ and $2 D$ cases, respectively. These methods are known to be stable. Results are presented for the comparison between the two models.
\end{abstract}

\section{Introduction}

Stochasticity plays an important role in cardiac myocyte calcium dynamics. Two main reasons given previously [1,2] are: 1 - The occurrence of sparks are random and spontaneous; 2 - The behaviour of calcium waves can differ significantly from deterministic to stochastic systems due to different levels of $\mathrm{Ca}^{2+}$ sensitivity of the calcium release units (CRUs) causing differences in the trigger of calcium waves.

A system of coupled PDEs, describing the cytosolic and sarcoplasmic $\mathrm{Ca}^{2+}$ concentrations has been used to simulate deterministic and stochastic waves [3]. Their model has three main drawbacks [4]: 1 - Triggering of $\mathrm{Ca}^{2+}$ release relies on a deterministic rule which occurs when the concentration exceeds some fixed value $C^{*} ; 2-$ Only isotropic diffusion of $\mathrm{Ca}^{2+}$ is considered, and $3-\mathrm{Ca}^{2+}$ buffers and dye are not included, with their absence compensated for by the 10 -fold reduction of the free $\mathrm{Ca}^{2+}$ diffusion coefficient. Shortly after this, a numerical model of calcium spark formation and detection was formulated [4]. This model simulates the effects of dye and buffers but neglects the concentration in the SR and preserves the deterministic rule for triggering release. Later, the model was reformulated to include a stochastic rule for the release of CRUs for the anisotropic diffusion of $\mathrm{Ca}^{2+}[1,5]$.

The models above use Fick's Law for the $\mathrm{Ca}^{2+}$ diffusion. An extension of the Fick's Law diffusion to anomalous subdiffusion has been reported to give more realistic results $[6,7,8]$. Since the work of Keizer and Smith (1998), models have focused on including buffers, stochastic calcium release and anomalous subdiffusion.

Except for one work [9], the coupled behaviour of the cytosolic and sarcoplasmic concentration has not been modelled. The model presented in this paper is a stochastic extension of the deterministically coupled model previously used by $[10,11]$, which includes the cytosolic leak and a representation of the SR leak not found in existing stochastic models. We compare simulation results for the 1D and 2D cases and present results for the anisotropic case of the 2D model.

\section{Methods}

\subsection{Model for calcium sparks and blinks}

It has been reported that there is little variation of calcium waves in the z-direction $[8,12]$. For this reason, this paper focuses on a $2 \mathrm{D}$ model by eliminating the gradients along the z-direction. A 1D simulation is also presented to compare model results.

The governing equations for $\mathrm{Ca}^{2+}$ sparks and blinks are based on the Fick's Law diffusion for simplicity

$$
\begin{gathered}
\frac{\partial C}{\partial t}=D_{C x} \frac{\partial^{2} C}{\partial x^{2}}+D_{C y} \frac{\partial^{2} C}{\partial y^{2}}+J_{\text {dye }}+J_{\text {buffer }}-J_{\text {pump }}- \\
J_{C a^{2+} \text { leak }}+J_{C R U}+J_{\text {SR leak }} \\
\frac{\partial C_{S}}{\partial t}=-J_{\text {SR leak }}+J_{\text {pump }}-J_{C R U}+J_{\text {dye }}+J_{\text {buffer }}
\end{gathered}
$$

where $C=\left[\mathrm{Ca}^{2+}\right]_{c y t(\infty)}$ and $C_{S}=\left[\mathrm{Ca}^{2+}\right]_{S R(\infty)}$ represent the free $\mathrm{Ca}^{2+}$ concentration in the cytosol and $\mathrm{SR}$, and $\mathrm{JCa}^{2+}{ }_{\text {leak }}$ is the cytosolic $\mathrm{Ca}^{2+}$ leak from to the exterior of the cell. The remaining terms are previously described $[7,8]$. The terms $J_{d y e}, J_{\text {buffer }}, J_{\text {pump }}, J_{C a^{2+} \text { leak }}$ and $J_{\text {SR leak }}$ are

$J_{\text {buffer }}=-\frac{\partial[\mathrm{CaB}]}{\partial t}$ 


\begin{tabular}{|c|c|c|}
\hline \multicolumn{3}{|c|}{$J_{d y e}=-k_{F}^{+} C\left([F]_{T}-[C a F]\right)+k_{F}^{-}[\mathrm{CaF}]$} \\
\hline \multirow{2}{*}{\multicolumn{3}{|c|}{$\begin{array}{l}\frac{\partial[C a B]}{\partial t}=k_{B}^{+} C\left([B]_{T}-[C a B]\right)-k_{B}^{-}[C a B] \\
\frac{\partial[C a F]}{=}=D_{0} \frac{\partial^{2} C a F}{d a}+D_{0} \frac{\partial^{2} C a F}{-}-\end{array}$}} \\
\hline & & \\
\hline \multicolumn{3}{|c|}{ 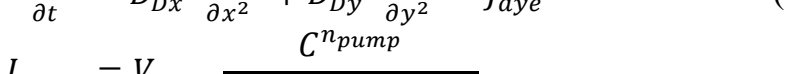 } \\
\hline \multicolumn{3}{|c|}{$J_{\text {pump }}=V_{\text {pump }} \overline{K_{\text {pump }}^{n_{\text {pump }}}+C^{n_{\text {pump }}}}$} \\
\hline \multirow{3}{*}{\multicolumn{3}{|c|}{$\begin{array}{l}J_{C a^{2+} \text { leak }}=k C \\
J_{S R \text { leak }}=k_{f} C_{s} \\
\text { where } k C \text { and } k_{f} C_{s} \text { refer to the passive efflux from th } \\
\text { cytosol and SR, respectively. Standard parameter value } \\
\text { are given in Tables } 1 \text { and } 2 .\end{array}$}} \\
\hline & & \\
\hline & & \\
\hline \multicolumn{3}{|c|}{ Table 1. Standard parameter values. } \\
\hline Parameter & Value & Source \\
\hline$D_{C x}, D_{C y}$ & $0.3,0.15 \mu \mathrm{m}^{2} / \mathrm{ms}$ & {$[1,5,7,8]$} \\
\hline$D_{D x}, D_{D y}$ & $0.02,0.01 \mu \mathrm{m}^{2} / \mathrm{ms}$ & {$[1,5,7,8]$} \\
\hline$l_{x}, l_{y}$ & $2,0.8 \mu \mathrm{m}$ & {$[1,5,7,8]$} \\
\hline$\left[\mathrm{Ca}^{2+}\right]_{c y t(\infty)}$ & $0.1 \mu \mathrm{M}$ & {$[1,5,7,8]$} \\
\hline$\left[\mathrm{Ca}^{2+}\right]_{S R(\infty)}$ & $750 \mu \mathrm{M}$ & [9] \\
\hline$F$ & $96500 \mathrm{C} \mathrm{mol}^{-1}$ & {$[1,5,7,8]$} \\
\hline$V_{\text {pump }}$ & $0.208 \mu \mathrm{M} / \mathrm{ms}$ & {$[1,5,7,8]$} \\
\hline$K_{\text {pump }}$ & $0.184 \mu \mathrm{M}$ & {$[1,5,7,8]$} \\
\hline$K_{\text {prob }}$ & $15 \mu \mathrm{M}$ & {$[1,5,8]$} \\
\hline$n_{\text {pump }}, n_{\text {prob }}$ & $3.9,1.6$ & {$[1,5,8]$} \\
\hline$T_{\text {open }}$ & $10 \mathrm{~ms}$ & {$[1,5,8]$} \\
\hline$P_{\max }$ & 0.3 per $\mathrm{CRU} \mathrm{ms}^{-1}$ & {$[1,5$} \\
\hline$\sigma_{3}$ & $I_{C R U} / 2 F \mathrm{~mole} / \mathrm{ms}$ & {$[1,5,7,8]$} \\
\hline$k$ & $0.012 \mathrm{~ms}^{-1}$ & \\
\hline$k_{f}$ & $0.00038 \mathrm{~ms}^{-1}$ & \\
\hline$I_{C R U}$ & $20 \mathrm{pA}$ & \\
\hline
\end{tabular}

The flux of calcium release from the CRUs is determined by $J_{C R U}$,

$J_{C R U}=\sum_{\alpha, \beta} \sigma \delta\left(x-x_{\alpha}\right) \delta\left(y-y_{\beta}\right) S\left(x, y, t ; T_{\text {open }}\right)$

where $\sigma$ is a molar flux from a CRU channel which is based on the CRU current, $I_{C R U}$, and Faraday's constant, F. The remaining terms can be found in previous models $[1,7,8]$.

The CRUs are placed at regular intervals of $l_{x}$ along Xaxis and $l_{y}$ along y-axis. It is assumed that once a CRU opens it will remain open for $10 \mathrm{~ms}$ (i.e. $T_{\text {open }}=10 \mathrm{~ms}$ ) after which it will switch to a closed state and will not reopen (a long refractory period).

Table 2. Standard parameter values for dye and endogenous buffer.

\begin{tabular}{lllll}
\hline Buffers & $k^{+}\left((\mu \mathrm{Ms})^{-1}\right)$ & $k^{-}\left(\mathrm{s}^{-1}\right)$ & {$[B]_{T}(\mu \mathrm{M})$} & Source \\
\hline Dye & 80 & 90 & 50 & {$[1,5,7,8]$} \\
Buffer & 100 & 100 & 123 & {$[5]$} \\
\hline
\end{tabular}

An adjustment to the source strength was previously done to approximate the molar flux of a point source [1]. This approach is extended here for the 1D case (See Appendix for the obtained $\sigma_{1}$ and $\sigma_{2}$ values).

\subsection{Numerical methods}

The computational domain used for all simulations has dimensions of $20 \times 100 \mu \mathrm{m}$ which is meshed with a grid size of $\frac{0.8}{3} \times 0.4 \mu \mathrm{m}$ and time step size of $0.003125 \mathrm{~ms}$.

The governing equations $(1,4)$ are discretised using the ADI method with standard finite difference notation. Therefore, Equation (1) becomes

$$
\begin{gathered}
\left(\frac{\Delta t}{2}\left(k-D_{C x} \frac{\delta_{x}^{2}}{\Delta x^{2}}\right)+1\right) C_{i, j}^{n+\frac{1}{2}}=\left(1+\frac{\Delta t}{2} D_{C y} \frac{\delta_{y}^{2}}{\Delta y^{2}}\right) C_{i, j}^{n}+ \\
\frac{\Delta t}{2}\left(J_{\text {dye }}+J_{\text {buffer }}-J_{\text {pump }}+J_{C R U}+J_{\text {SR leak }}\right) \\
\left(\frac{\Delta t}{2}\left(k-D_{C y} \frac{\delta_{y}^{2}}{\Delta y^{2}}\right)+1\right) C_{i, j}^{n+1}=\left(1+\frac{\Delta t}{2} D_{C x} \frac{\delta_{x}^{2}}{\Delta x^{2}}\right) C_{i, j}^{n+\frac{1}{2}}+ \\
\frac{\Delta t}{2}\left(J_{\text {dye }}+J_{\text {buffer }}-J_{\text {pump }}+J_{C R U}+J_{\text {SR leak }}\right)
\end{gathered}
$$

Similarly, equation (4) becomes

$$
\begin{aligned}
\left(1-\frac{\Delta t}{2} D_{D x} \frac{\delta_{x}^{2}}{\Delta x^{2}}\right)[C a F]_{i, j}^{n+\frac{1}{2}} & =(1+ \\
\frac{\Delta t}{2} & \left.D_{D y} \frac{\delta_{y}^{2}}{\Delta y^{2}}\right)[C a F]_{i, j}^{n}-\frac{\Delta t}{2} J_{d y e} \\
\left(1-\frac{\Delta t}{2} D_{D y} \frac{\delta_{y}^{2}}{\Delta y^{2}}\right)[C a F]_{i, j}^{n+1} & =(1+ \\
\frac{\Delta t}{2} & \left.D_{D x} \frac{\delta_{x}^{2}}{\Delta x^{2}}\right)[C a F]_{i, j}^{n+\frac{1}{2}}-\frac{\Delta t}{2} J_{d y e}
\end{aligned}
$$

Simple impermeability is assumed for the cell boundaries leading to Neumann conditions $\partial C /$ $\left.\partial n\right|_{\text {boundary }}=0$ on all edges. The models are run for $1 \mathrm{~s}$.

For the 1D case, a CN Finite Difference approximation is used to discretise the governing equations $(1,4)$. In both the $1 \mathrm{D}$ and $2 \mathrm{D}$ cases, equations $(2,3)$ are solved using the forward Euler method. Standard parameter values given in Tables 1 and 2 are used unless otherwise stated.

\section{Results}

\subsection{Comparison of the $1 \mathrm{D}$ and $2 \mathrm{D}$ models}

The 2D model is set to be isotropic $\left(D_{C x}=D_{C y}=0.2\right.$ and $D_{D x}=D_{D y}=0.02$ ) with two columns of point sources in the middle of the domain. The source strengths used are $\sigma_{1}=0.19 \sigma_{3}$ and $\sigma_{2}=0.51 \sigma_{3}$ (Appendix). The results obtained are given in Figure 1. The amplitude of the cytosolic calcium concentration (A,C) slightly varies, while a much greater variation is seen in the sarcoplasmic calcium concentration (B,D).

Another 2D study was performed, replacing the two columns of point sources with continuous lines of CRUs with the same source strength as that of the 1D case. The $1 \mathrm{D}$ and $2 \mathrm{D}$ results were identical. This was an important observation indicating that the large difference in SR calcium blink amplitude from the 2D to the $1 \mathrm{D}$ case is due to the spacing between the channels in the transverse direction. The dimension reduction also leads to missing characteristics from 2D to 1D in the terms associated with 
the diffusion (in the y-axis) and calcium source.
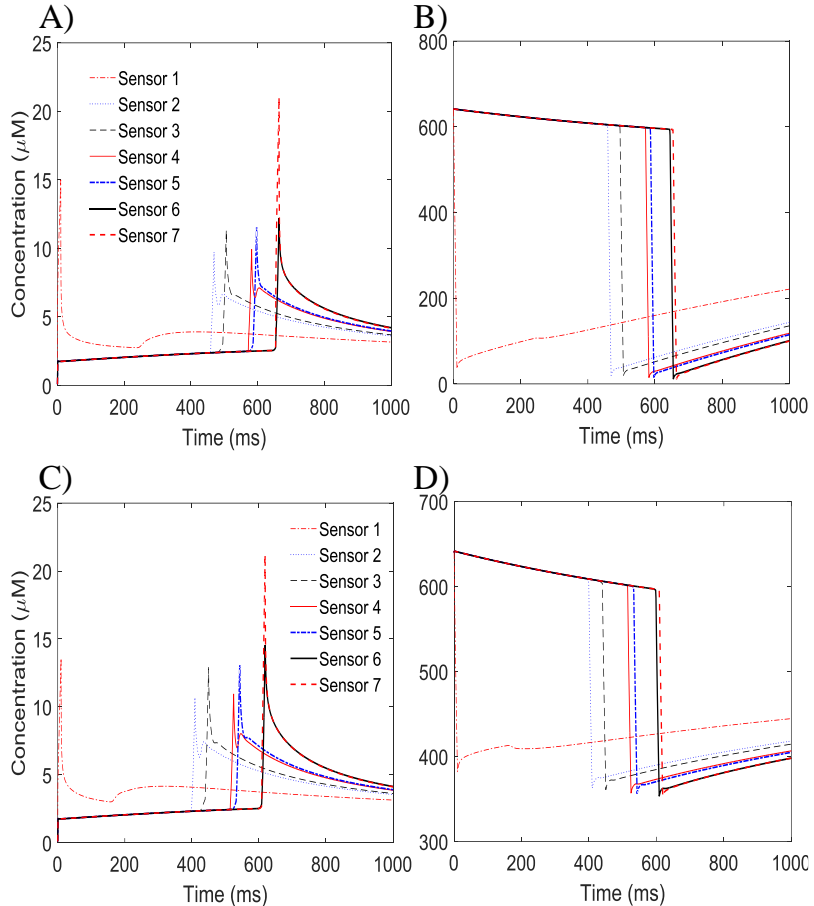

D)

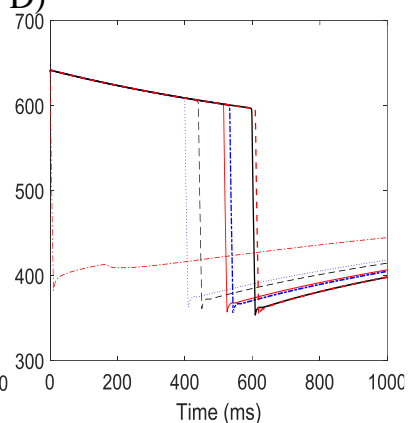

Figure 1. 2D model results (A,B) and 1D model results (C,D) for cytosolic $(\mathrm{A}, \mathrm{C})$ and sarcoplasmic $(\mathrm{B}, \mathrm{D})$ calcium concentration in time. Sensors 1-7 are located at 50, 25, 75, $12,88,99$ and $100 \mu \mathrm{m}$ longitudinally and $9.6 \mu \mathrm{m}$ in the transverse direction for the 2D case. $\left[\mathrm{Ca}^{2+}\right]_{S R(\infty)}=640 \mu \mathrm{M}, k_{f}=0.00042 \mathrm{~ms}^{-1}$.

\subsection{D model: anisotropic diffusion}

Figure 2 shows the propagation of $\mathrm{Ca}^{2+}$ wave on a rectangular domain. The calcium concentration ranges between $0.1 \mu \mathrm{M}$ and $23 \mu \mathrm{M}$. The calcium wave is initiated from the middle of the domain where $3 \times 9$ CRUs are activated. Once activated the release lasts for $10 \mathrm{~ms}$ in which it reaches approximately $16-18 \mu \mathrm{M}$ (Figure 3.A,B). As the concentration increases, the probability of CRUs firing increases. The succession of releases forms a calcium wave as seen in Figure 2.

Figure 3 shows the time courses of the concentration in the cytosol and SR. Calcium sparks in the cytosol leads to a calcium blinks in the SR calcium concentration of $\sim 4$ fold of the cytosolic concentration. In this model, a middle slice at $9.6 \mu \mathrm{m}(\mathrm{A}, \mathrm{B})$ and near the upper edge $19.2 \mu \mathrm{m}$ $(\mathrm{C}, \mathrm{D})$ are observed. The sparks and blinks at the upper edge are observed to be slightly higher for cytosolic and slightly less for sarcoplasmic compare to that of the middle. This is expected as the concentration increases at the boundaries [8]. In this particular simulation, a longitudinal velocity of $v_{x}=116 \mu \mathrm{m} / \mathrm{s}$ was observed which is close to a previously reported value [8] and slightly above the physiological value $(100 \mu \mathrm{m} / \mathrm{s})$.
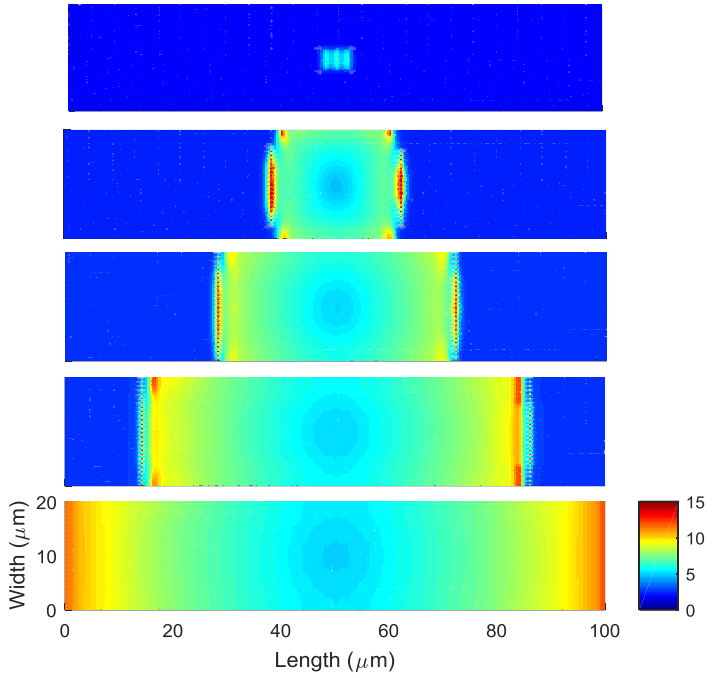

Figure 2. Snapshots of the propagation of cytosolic calcium for times 12.5, 212.5, 287.5, 362.5, $425 \mathrm{~ms}$ (from top to bottom). Values on colourbar is in $\mu \mathrm{M}$.

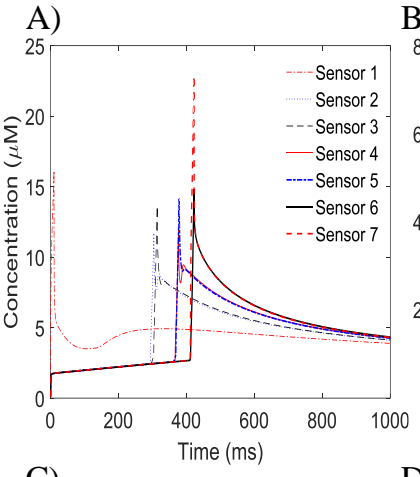

C)

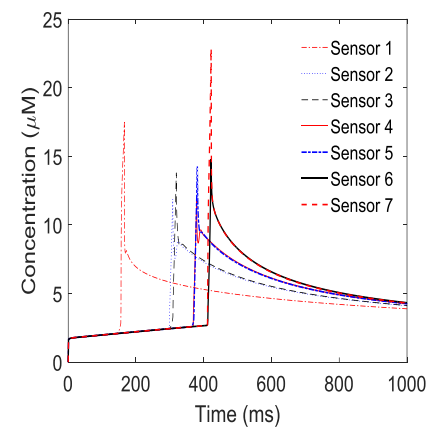

B)

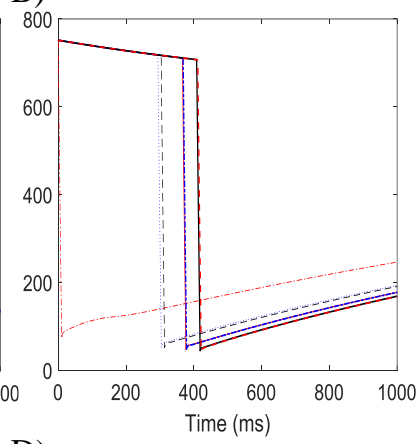

D)

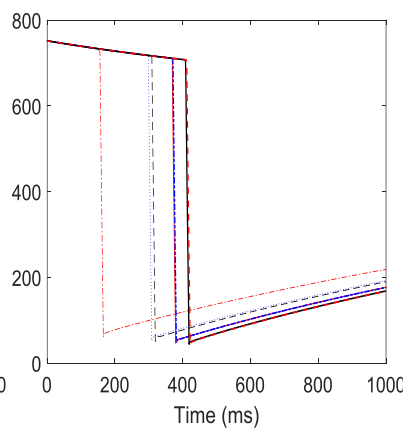

Figure 3. Time courses of cytosolic (A,C) and sarcoplasmic $(\mathrm{B}, \mathrm{D})$ calcium concentrations at the middle $(A, B)$ and upper edge $(19.2 \mu \mathrm{m})$ of the domain.

The time course of $\mathrm{F} / \mathrm{F}_{0}$ from the cytosolic calcium profiles peaks at approximately 2.6 and shows a pattern previously observed in experimental $[2,13,14,15]$ and numerical studies $[6,14,15]$. The normalized spatial profiles of $\mathrm{CaF}$ is known to have full width at half maximum (FWHM) of $1 \mu \mathrm{m}$ for diffusion based on Fick's law. Here a FWHM of approximately $0.7 \mu \mathrm{m}$ is observed (Figure 4 right). 

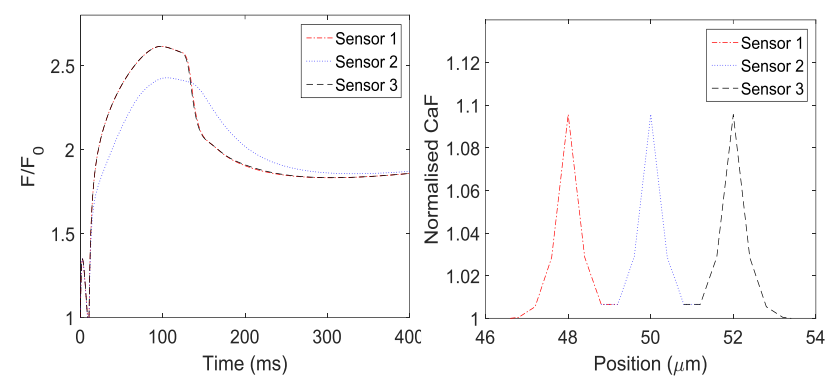

Figure 4. $F / F_{0}$ indicator dye in time (left) and a spatial plot of the normalised $\mathrm{CaF}$ (right).

\section{Discussion and conclusion}

An anisotropic calcium diffusion model based on the two-pool model was presented. Comparison between the $1 \mathrm{D}$ and $2 \mathrm{D}$ results reveal that spacing between channels along the $y$-axis trigger a large variation in the SR calcium concentration. Further research is needed to clarify the level of variation. This also leads us to question whether spacing between channels along the z-axis also introduces a large variation in the SR calcium concentration.

Results from the anisotropic diffusion model were also presented, where the behavior of the cytosolic and sarcoplasmic calcium was observed. The patterns observed as well as $\mathrm{F} / \mathrm{F}_{0}$ and normalized $\mathrm{CaF}$ were as expected. The model for the SR calcium concentration requires further research to investigate the variation noted earlier as well as the effects of varying the duration of blinks on the behaviour of calcium sparks and blinks.

\section{Appendix}

The numerical conversion for the source strength involves the Green's function obtained from the analytical solution of the diffusion problem for each of the dimensional cases and convolving this with the Heaviside function. Some description on the derivation is given previously [1]. The deduced relations for each case are

$$
\begin{aligned}
& C(r, t, 3)=\sigma_{3} /(4 \pi w) \operatorname{erf}\left(\frac{w}{2 \sqrt{t}}\right) \\
& C(r, t, 2)=\sigma_{2} /(4 \pi D) E_{i}\left(\frac{D_{C x} y^{2}+D_{C y} x^{2}}{4 D_{C x} D_{C y} t}\right) \\
& C(r, t, 1)=\sigma_{1} /(2 D \sqrt{\pi})\left(2 D \sqrt{t} \exp \left(-\frac{x^{2}}{D^{2} t}\right)^{0.25}\right. \\
& \left.+\sqrt{\pi} \operatorname{erf}\left(\frac{x}{2 D \sqrt{t}}\right)\right)
\end{aligned}
$$

where $w=\sqrt{\left(D_{C x} y^{2}+D_{C y} x^{2}\right) D_{C z}+z^{2} D_{C x} D_{C y}}$ and $D$ is the geometric mean of $D_{C x}, D_{C y}$ and $D_{C Z}$. Sigma values in section 3.1 are $\sigma_{1}=0.19 \sigma_{3} / \mu \mathrm{m}^{2}$, and $\sigma_{2}=0.51 \sigma_{3} / \mu \mathrm{m}$. For the anisotropic case (section 3.2) a sigma value of $\sigma_{2}=$ $0.59 \sigma_{3} / \mu \mathrm{m}$ is used.

\section{Acknowledgements}

This work was supported by a VC scholarship of the Greenwich University (Ref. No: VCS-ACH-15-14). The authors would like to deeply thank fellow researcher, Christopher Beckwith, for his invaluable thoughts on the comparison results that led to important observations.

\section{References}

[1] Izu L.T., Wier W.G., Balke C.W. Evolution of cardiac calcium waves from stochastic calcium sparks. Biophys $\mathbf{J}$ 2001;80:103-120.

[2] Cheng H., Lederer W.J., Cannell M.B. Calcium sparks: elementary events underlying excitation-contraction coupling in heart muscle. Science 1993;262:740-744.

[3] Keizer J., Smith G.D. Spark to wave transition: salutatory transmission of calcium waves in cardiac myocytes. Biophys Chem 1998;72:87-100.

[4] Izu L.T., Wier W.G., Balke C.W. Theoretical analysis of the $\mathrm{Ca}^{2+}$ spark amplitude distribution. Biophys J 1998;75:11441162.

[5] Izu L.T., Mauban J.R.H., Balke C.W., Wier W.G. Large currents generate cardiac $\mathrm{Ca}^{2+}$ sparks. Biophys J 2001;80:88102.

[6] Li K., Fu C., Cheng H., Tan W. Anomalous subdiffusion of calcium spark in cardiac myocytes. Cell Mol Bioeng 2011;4:457-465.

[7] Chen X., Kang J., Fu C.., Tan W. Modeling calcium wave based on anomalous subdiffusion of calcium sparks in cardiac myocytes. PLoS One 2013;8:1-9.

[8] Chen X., Guo L., Kang J., Huo Y., Wang S., Tan W. Calcium waves initiating from the anomalous subdiffusive calcium sparks. J R Soc 2014;11:1-10.

[9] Li J., Xie W., Chen X., Huo Y., Cheng H., Tan W. A novel stochastic reaction-diffusion model of $\mathrm{Ca}^{2+}$ blink in cardiac myocytes. Sci Bull 2017;62:5-8.

[10] Dupont G., Goldbeter A. Properties of intracellular $\mathrm{Ca}^{2+}$ waves generated by a model based on $\mathrm{Ca}^{2+}$-induced $\mathrm{Ca}^{2+}$ release. Biophys J 1994;67:2191-2204.

[11] Dupont G., Goldbeter A. Modelling oscillations and waves of cytosolic calcium. Non Anal, Theory, Meth Appl 1997;30:1781-1792.

[12] Ishida H., Genka C., Hirota Y., Nakazawa H., Barry W.H. Formation of planar and spiral $\mathrm{Ca}^{2+}$ waves in isolated cardiac myocytes. Biophys J 1999;77:2114-2122.

[13] Cheng H., Lederer M.R., Xiao R-P., Gomez A.M., Zhou YY., Ziman B., Spurgeon H., Lakatta E.G., Lederer W.J. Excitation-contraction coupling in heart: new insights from $\mathrm{Ca}^{2+}$ sparks. Cell Calcium 1996;20:129-140.

[14] Soeller C., Cannell M.B. Estimation of the sarcoplasmic reticulum $\mathrm{Ca}^{2+}$ release flux underlying $\mathrm{Ca}^{2+}$ sparks. Biophys J 2002;82:2396-2414.

[15] Bers D.M., Channon T.R. Calcium movement inside the sarcoplasmic reticulum of cardiac myocytes. J Mol Cell Cardiol 2013;58:59-66.

Address for correspondence.

Serife Arif

30 Park Row, University of Greenwich

London, UK, SE10 9LS

(s.arif@greenwich.ac.uk) 